\title{
Some effects of Vowel Space Area (VSA) reduction in speech intelligibility
}

Patrizia Sorianello

Department of Letters, Languages and Arts. University of Bari, Italy

https://doi.org/10.36505/ExLing-2015/06/0020/000257

\begin{abstract}
This study intends to examine the correlation between the size of vowel space and the speech intelligibility. A reduced intelligibility is a common problem for those individuals suffering from dysarthria. They show a compressed vowel space with an important degree of acoustic centralization. A recent metric employed to distinguish healthy from dysarthric speech is represented by the Vowel Space Area (VSA). We have tested this parameter by comparing subjects with Down Syndrome to control speakers. The results of the present research, the first available data for Italian, showed that the VSA is a suitable acoustic predictor of an impaired intelligibility.
\end{abstract}

Key words: speech intelligibility, Vowel Space Area, Down Syndrome, dysarthria

\section{Introduction}

Intelligibility refers to the degree of comprehensibility of speech. A reduced intelligibility is an aspect of speech disorders, irrespective of the underlying neurological condition. Most types of dysarthria are characterized by the loss of vowel clarity, altered range of articulatory movements and vowel centralization. The methodological procedures used to estimate the intelligibility include parental surveys, tests based on the auditive identification of words and scoring from transcriptions. Previous studies have shown a positive relationship between the size of vowel system and the overall intelligibility (Weismer et al 2001, Liu et al. 2005). Larger vowel space are expected to be associated in more intelligible subjects, while smaller vocalic system are expected for dysarthric ones. Vowel Space Area (VSA) is an acoustic metric able to distinguish dysarthric patients from healthy control ones. VSA is constructed by the F1/F2 values of the three corner vowels $/ \mathrm{i} /, / \mathrm{a} /, / \mathrm{u} /$ and calculated in accordance with the equation (1):

$$
\text { (1) triangular VSA: [(F1i (F2a-F2u) + F1a (F2u-F2i) + F1u (F2i-F2a) }] / 2
$$

Nevertheless, the predictive force of VSA is discussed, since sometimes it failed. The VSA seems to depend on several external factors such as the degree of inter-speaker variability or the number of the vowels considered. For this reason, in recent studies other metrics have been also considered (for a review, Lansford \& Liss, 2014).

ExLing 2015: Proceedings of 6th Tutorial and Research Workshop on Experimental Linguistics, 26-27 June 2015, Athens, Greece 


\section{Speech intelligibility in Down Syndrome}

In this research we focus our attention on individuals with Down Syndrome (DS). They exhibit an atypical language with a degraded intelligibility. Generally, the speech of people with (DS) is difficult to understand: phonetic alterations, dysfluency (stuttering or cluttering), altered voice quality and dysprosody are the most frequent manifestations of an impaired intelligibility (among all, Nadel 1998, and for Italian language Sorianello 2012). In DS the precision of the articulatory gestures involved in the phonation is altered by several structural restrictions such as facial hypoplasia, hearing difficulties, breathing dysfunctions, memory deficits. In addition, speech intelligibility is also affected by impairment in both oral motor control and planning (Kumin et al. 2006). With regard to the vowels, the presence of a smaller vowel space has been observed in DS as compared to matched control group (Moura et al. 2008, Bunton \& Leddy 2011). Speech intelligibility of Italian people with DS has never been instrumentally analyzed. The aim of the present study was to examine the effects of a reduced speech intelligibility on vowel working space.

\section{Methodology}

Two groups of Italian subjects took part in this study. Ten young adults with DS ( $5 \mathrm{M}, 5 \mathrm{~F})$, aged between 16 and 22 and lived in Cosenza, a city of Southern Italy, were compared to age-matched controls (3 M, $3 \mathrm{~F}$ ) (CG). A corpus of 1200 stressed vowels was analyzed. It consisted of Isolated Words; subjects were asked to named 108 pictures presented on cards representing common objects or actions. All participants were audio recorded using a digital TASCAM DR07; for people with DS we previously obtained informant consent from the family. For each vowel the following parameters were extracted by means of the software PRAAT: 1) F1, F2 frequency values, 2) Centroid value, 3) triangular VSA, 4) coefficient of variation (CV: Standard deviation/mean). The formants were manually extracted using FFT procedure, at the correspondence of the steady-state portion of the formant trajectory. A one-way Anova test was performed.

\section{Results}

In Figures 1-2 the VSA of DS and CG subjects are graphically shown; the mean values of the VSA are listed in Table 1 . In DS the vowel space is relatively small, above all in males. As expected (see Table 1), the mean values of the VSA are lower in males than females, in both groups. Compared to control speakers, the VSA size of subjects with DS is always smaller; a significant group effect (DS vs. CG) is found ( $p=.001)$. In detail, in males the size of VSA is $61 \%$ smaller with respect to the CG, whereas in females the difference amounts to $44,5 \%$ (males vs. females $\mathrm{p}=.001$ ). Data are consistent with a condition of 
altered speech intelligibility, suggesting that the VSA is a valid metric to capture differences between groups.
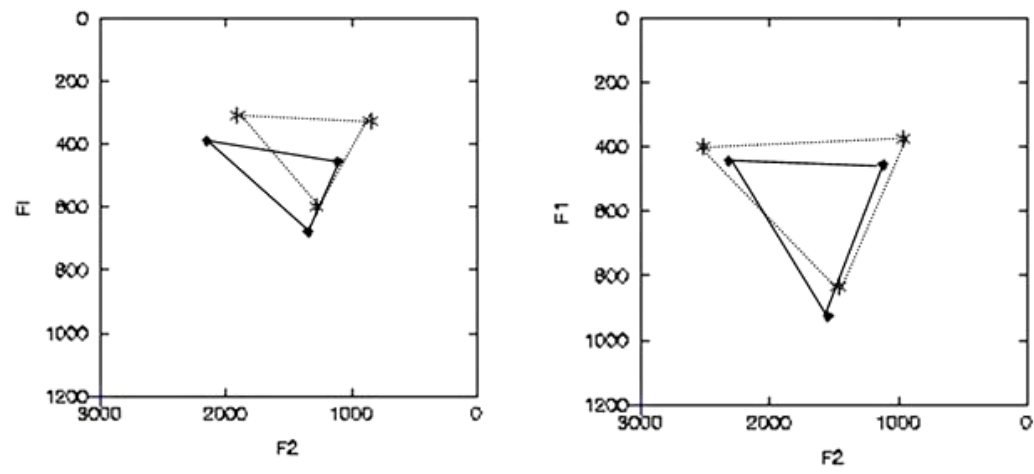

Figure 1. VSA of individuals with DS (closed line) and CG individuals (dashed line), grouped into males (left) and females (right)

Table 1. Mean, standard deviation (SD) and coefficient of variation (CV) of the triangular VSA for DS and CG. VSA and SD are expressed in Hz2.

\begin{tabular}{|c|c|c|c|c|}
\hline \hline \multicolumn{5}{|c|}{ Dowel Space Area } \\
\hline Values & Dales & females & males & females \\
\hline Mean & 125.144 & 240.161 & 201.508 & 347.052 \\
\hline SD & 12.171 & 55.779 & 54.452 & 38.446 \\
\hline CV & $9.7 \%$ & $23.2 \%$ & $27 \%$ & $11,1 \%$ \\
\hline \hline
\end{tabular}

The coefficient of variation is less informative, being lower in males with DS, but relatively large in females. The degree of vowel dispersion is likely due to a certain inter-speaker variability. Overall, in DS the dispersion of front vowels is normally larger than that of back ones, due to the difficulties encountered by speakers with DS in establishing accurate lingual contacts.

\section{Conclusions}

Experimental results demonstrate that speakers with DS are effectively distinguished from healthy ones by means of the triangular VSA. There is a strong relationship between VSA and vowel distinctiveness: the smaller the area, the less intelligible the speech. So, at least in our data, the VSA is able of differentiating people with or without dysarthria and can be taken as a quantifier of vocal abnormality. Contrary to what was observed in other studies, an impaired intelligibility is anchored to the presence of a restricted VSA. This result can probably be attributed to the fact that all participants with DS show a 
significant level of dysarthria. People with DS meet hard difficulties in performing speech sounds. Their anatomical anomalies have strong implications on language production, subjects with DS have a more reduced F1/F2 plane than CG individuals. The presence of a large tongue, in relation to a smaller oral cavity, produces inadequate movements of tongue, lips and jaw. The inability to synchronise oral gestures is the cause of frequent processes of phonological target undershoot. Moreover, the weakness of the oro-facial muscles, a lack of laryngeal control and motor disorder inevitably diminishes the accuracy of the speech sounds. Given this starting situation, it is not surprising that in DS the precision in achieving a specific articulatory target is strongly reduced. Their vocalic system shows a widespread dispersion: all the vowels share common areas. Very often, the vowels are too close within the system, so their articulatory identity becomes unclear and that interferes with the possibility to establish effective perceptive contrasts. From a phonetic point of view, a great deal of stressed vowels are indistinct sounds. This situation induces a consistent compression of the vowel space with clear vowel alterations. As a consequence, when the articulation is damaged, speech intelligibility automatically decays. In order to confirm our findings, in the future we intend to perform a more complete assessment of vowel articulation, exploring the impaired intelligibility by means of other metrics, such as the Formant Centralization Ratio (FCR) or the Vowel Articulation Index (VAI).

\section{References}

Bunton, K. and Leddy, M. 2011. An evaluation of articulatory working speech area in vowel production of adults with Down syndrome. Clinical Linguistics and Phonetics 25, 321-334.

Kumin, L. 2006. Speech intelligibility and childhood verbal apraxia in children with Down Syndrome. Down Syndrome Research and Practice 10, 10-22.

Lansford, K.L. and Liss, J.M. 2014. Vowel acoustics in dysarthria: speech disorder diagnosis and classification. Journal of Speech, Language and Hearing Research 57, 57-67.

Liu, H.M., Tsao, F.M. and Kuhl, P.K. 2005. The effect of reduced vowel working space on speech intelligibility in Mandarin-speaking young adults with cerebral palsy. Journal of Acoustical Society of America 117, 3879-3889.

Moura, C.P., Cunha, L, Vilarinho, H., Cunha, M., Freitas, et al., M. 2008.Voice parameters in children with Down Syndrome. Journal of Voice 22, 34-42.

Nadel, L. (ed.). 1988. The psychobiology of Down syndrome: issues in the biology of language and cognition, Cambridge (Mass.), MIT Press.

Sorianello, P. 2012. Linguaggio e Sindrome di Down, Milano, FrancoAngeli.

Weismer, G., Jeng, J.Y., Laures, J., Kent, R. and Kent, J. 2001. Acoustic and intelligibility characteristics of sentence production in neurogenic speech disorders. Folia Phoniatrica et Logopaedica 53, 1-18. 